(C) Masson, Paris, 1979.

Annales de Parasitologie (Paris)

1979 , t. $54, \mathrm{n}^{\circ} 6$, pp. $593-614$

\title{
Variations du système sensoriel de la cercaire de Schistosoma mansoni.
}

\author{
Intérêt éventuel en épidémiologie
}

\author{
par Ch. BAYSSADE-DUFOUR \\ Laboratoire de Zoologie (Vers), associé au C.N.R.S., Muséum national d'Histoire naturelle, \\ 43, rue Cuvier, 75231 Paris Cedex 05.
}

\section{Résumée.}

La bilharziose intestinale humaine provoquée par Schistosoma mansoni est une maladie africaine introduite depuis trois siècles environ en Amérique du Sud et aux Caraïbes.

Les soies antéacétabulaires latérales et les soies caudales préfurcales de la cercaire permettent de différencier les souches africaines des souches américaines.

L'établissement d'un indice portant sur les distances relatives entre certaines soies antéacétabulaires est susceptible de fournir des données objectives sur le difficile problème posé par l'adaptation des souches humaines aux Rats. L'adaptation d'un souche humaine aux Muridés ne s'effectue pas par une sélection brutale au cours du premier passage, comme il est habituel pour la plupart des parasites, mais par un phénomène complexe ne commençant à se manifester de façon décisive qu'au cours du quatrième passage environ ; expérimentalement, l'indice cercarien humain $(n=1,0)$ ne change pas lors des premier et deuxième passage, puis commence à se modifier au troisième passage $(n=1,3)$ et atteint 1,5 au quatrième passage. Cette notion d'adaptation tardive se retrouve dans l'étude de différents phénomènes observés par nous-mêmes ou nos collègues (soies du miracidium, réussite des passages expérimentaux, durée de la prépatence, traversée de la peau, etc...).

L'indice des parasites adaptés au Rat $(1,7)$, très différent de l'indice des parasites adaptés à l'Homme $(1,0)$ nous parait avoir une très grande importance pratique pour deux problèmes essentiels dans la bilharziose intestinale.

a. Gravité des formes cliniques. On peut supposer que les souches murines seraient à l'origine des formes très graves de la maladie (cirrhose portale), car elles seraient mal adaptées à l'Homme. Il devient possible, par la simple détermination de l'indice cercarien des parasites émis par chaque malade, de voir s'il y a une corrélation entre la gravité clinique et l'indice plus ou moins élevé des cercaires, et d'infirmer ou de confirmer cette hypothèse.

Accepté le 13 novembre 1979. 
b. Existence de foyers murins. Une seconde question, très importante, qui se pose à propos de cette maladie, est de savoir si elle peut se maintenir spontanément chez l'animal, endehors de toute contamination humaine.

En Guadeloupe, les nombreuses souches des différentes régions de l'île, provenant soit du Mollusque, soit du Rat, soit de l'Homme, ont un indice d'environ 1,3, différent de l'indice 1,0 d'Afrique. Cet indice pourrait provenir de l'adaptation à un Mollusque différent (B. glabrata au lieu de B. pfeifferi), mais nous pensons plutôt qu'il s'agit d'un morphe indiquant l'existence de souches passant alternativement chez le Rat et chez l'Homme. A ces données s'opposent celles d'un gîte bien particulier (celui de Grand-Etang), où l'indice cerca. rien, égal à 1,6, est comparable à celui des souches de laboratoire entretenues depuis de nom. breuses années chez les Muridés. Nous croyons donc pouvoir confirmer les données épidémiologiques de Combes, Léger et Golvan, 1975, qui concluaient à l'existence d'une maladie purement murine dans ce biotope particulier.

\section{Summary.}

The variability in the pattern of sensory receptors in cercaria of Schistosoma mansoni and its possible relation to epidemiology of bilharziasis.

It is generally accepted that human intestinal bilharziasis due to Schistosoma mansoni originated in Africa and was introduced into South America and the West-Indies about three centuries ago.

A study of the chaetotaxy of the cercariae as well as experimental breeding showed that there are small differences between the African and American strains which can be considered infraspecific: lateral preacetabular setae are in a triangular pattern in the African strains and along a line in the American strains and there are four or five prefurcal caudal dorsal and ventral setae in the African, and six or more in the American strains. This enables the African and the American strains to be distinguished.

Chaetotaxy can also throw some light on the processes leading to adaptation of Schistosoma mansoni to murine-rodents. The cercarial index (c.i.) differs between the pure human and pure murine strains. The cercarial index is the ratio of a distance between the first and second lateral preacetabular setae to the distance between the first and second dorsal preacetabular setae. The cercarial index of the human African strains $(c . i .=1.00)$ differs from the c.i. of the human American strains (1.10 to 1.40) and from the murine welladapted parasites (1.69 to 2.34 ).

Experimental infection showed that the morphological adaptation of the human strain to murine rodents is a gradual process that begins only after the third passage in murines. This observation of a slow adaptation was also noted in a change of number of miracidial setae, snail susceptibility to infection, the production of cercariae, the length of the prepatent period, as well as the ability of the different cercariae to penetrate murine of human skin.

The cercarial index may be of practical importance in the understanding of clinical and epidemiological problems of bilharziasis :

a. Severity of disease. One could speculate that cases of severe bilharziasis could perhaps be caused by murine strains poorly adapted to Man. It is now possible by a single determination of the cercarial index to establish the origin of the infestation.

b. Epidemiological significance of chaetotaxy. An important problem raised by this disease is to know if the parasite population can persist in animals without any human contamination. 
Numerous strains of S. mansoni were isolated from different villages of Guadeloupe, from naturally infected snails, naturally infected rats and human cases. The cercarial index in all these Guadeloupean strains was on the average 1.30 (1.10 to 1.40$)$ as compared to the average c.i. of 1.00 in the African strains. The cercarial index of 1.30 may result from an adaptation to a different intermediate host (Biomphalaria glabrata instead of B. pfeifferi) but we believe it more probable that in Guadeloupe a form of $S$. mansoni exists having strains passing alternately in rats and in men.

It is interesting to note that a strain with a cercarial index of 1.63 was found in an isolated lake in a mountainous region of Guadeloupe, far from any village or cultivated field (Grand-Etang) where S. mansoni occurs only in rats. This index is similar to the c.i. of strains maintained for a long time in laboratory murine hosts.

This finding and hypothesis corroborate the epidemiologic data of Combes, Léger et Golvan, 1975 who concluded that an exclusively murine strain exists in this locality.

L'étude de l'appareil sensoriel, chez les Invertébrés, procure souvent des renseignements d'ordre systématique d'une très grande précision. Les travaux de J. Richard, 1971, ont montré son très grand intérêt chez les cercaires de Trématodes. L'appareil sensoriel des cercaires constitué essentiellement de papilles, s'imprègne par le nitrate d'argent selon trois techniques légèrement différentes : celle de Chatton et Lwoff, 1930, celle de Lynch, 1933 et, celle que nous avons utilisée, de Combes, Bayssade-Dufour et Cassone, 1976. L'utilisation de ces techniques a montré, dans le cas de Schistosoma mansoni, agent de la bilharziose intestinale des différences appréciables selon les souches: souches africaines ou américaines, souches d'origine humaine ou souches entretenues sur Muridés. Dans le cadre des recherches sur l'épidémiologie de la bilharziose, menées par le Pr Y.J. Golvan en Guadeloupe, l'objet de ce travail a été de savoir dans quelle mesure ces précisions morphologiques peuvent nous aider à résoudre deux problèmes essentiels.

- La maladie peut-elle se conserver dans des foyers strictement murins, sans l'intervention de l'Homme?

- Quel est le pouvoir pathogène pour l'Homme des souches bien adaptées aux Muridés?

\section{Variations selon le mollusque - hôte}

\section{1. - Soies latérales corporelles et soies préfurcales.}

La chétotaxie permet de distinguer les cercaires de souches africaines passant par Biomphalaria pfeifferi des cercaires de souches américaines passant par B. glabrata (Ch. Bayssade-Dufour, 1977 a).

a. SOUCHES ÉTUdiÉEs.

- Souches africaines. Trois jeunes B. pfeifferi sont infestés avec les miracidiums provenant des selles d'un malade ayant séjourné en Guinée et en République 
Centrafricaine; les cercaires correspondantes constituent le lot Guinée-R.C.A. Un lot de cercaires du Sénégal provient d'un Biomphalaria pfeifferi gaudi naturellement infesté, récolté à Fanden, dans la région de Thiès. Un lot de cercaires de Madagascar provient de quatre $B$. pfeifferi naturellement infestés, récoltés dans le district de Soavinandriana. Un lot de cercaires de Haute-Volta émises par douze B. pfeifferi provient d'un laboratoire qui entretient sur cette espèce de mollusques et sur le Singe Erythrocebus patas une souche de $S$. mansoni initialement isolée à partir d'un malade.

- Souches américaines. Une souche de Martinique est obtenue par l'infestation d'un jeune Biomphalaria glabrata sain, avec les selles d'un malade bilharzien. Un lot de Guadeloupe (Guadeloupe I) provient de six B. gabrata naturellement infestés, récoltés en Basse-Terre dans un canal d'irrigation, à "La Retraite ». Un autre lot de Guadeloupe a pour origine des mollusques récoltés à Pointe-à-Pître (Guadeloupe II); il a subi en laboratoire deux passages consécutifs sur Souris blanche et B. glabrata. Une souche originaire de Porto-Rico, entretenue depuis de nombreuses années sur B. glabrata et Souris blanche, procure des cercaires émises par deux mollusques infestés en laboratoire. Une souche originaire de Recife, au Brésil, entretenue depuis de nombreuses années sur B. glabrata et Souris blanche, fournit un lot de cercaires issues de dix mollusques infestés en laboratoire.

Pour chacune de ces neuf souches, nous avons imprégné au nitrate d'argent et observé une centaine de cercaires, sauf pour celle de Madagascar, où nous avons observé une vingtaine de cercaires seulement.

\section{b. Résultats.}

Les résultats observés concordent avec ceux donnés par J. Richard, 1971, et Short et Cartrett, 1973, sur la stabilité de certains groupes de papilles et la variabilité d'autres groupes. Toutefois, nous avons observé qu'il est possible de distinguer un type africain de Schistosoma mansoni, légèrement différent du type américain.

Les caractères différentiels sont les suivants :

- les papilles latérales: les papilles $\mathrm{A}_{1} \mathrm{~L}$ et $\mathrm{A}_{\mathrm{H}} \mathrm{L}$ sont disposées en triangle chez la majorité des cercaires africaines (fig. 1, col. 1); leurs homologues sont alignées chez la plupart des cercaires américaines (fig. 1, col. 4);

- les papilles préfurcales ventrales: sur le tronc caudal, après un groupe de six à sept papilles à localisation constante, nous observons deux papilles préfurcales ventrales chez la majorité des cercaires africaines (fig. 1, col. 2), trois papilles, ou parfois quatre chez la majorité des cercaires d'Amérique (fig. 1, col. 5);

- les papilles préfurcales dorsales: de même, après le groupe de six papilles dorsales à localisation constante, nous observons deux papilles préfurcales dorsales chez la plupart des souches africaines (fig. 1, col. 3), trois ou quatre papilles chez la plupart des cercaires américaines (fig. 1, col. 6). 


\begin{tabular}{|c|c|c|c|c|c|c|}
\hline & $\begin{array}{lll}6 & 0 \\
0 & 0 & 0 \\
0 & 0 & 0 \\
0 & 0 & 0 \\
0 & 0 & 0 \\
0 & 0 & 0\end{array}$ & $\begin{array}{r}30 \\
0 \\
0 \\
0 \\
0 \\
0 \\
0 \\
0 \\
0 \\
0 \\
\cdots\end{array}$ & (3) & $\begin{array}{ccc}\beta_{0} & & 0 \\
0 & \cdots & 0 \\
0 & \vdots & 0 \\
0 & 0 & 0 \\
0 & 0 & 0\end{array}$ & $\begin{array}{c}2 \\
0 \\
\therefore \\
0 \\
0 \\
0 \\
3 \\
0 \\
0\end{array}$ & 20 \\
\hline $\begin{array}{l}\text { GUINEE } \\
\text { RC A }\end{array}$ & $81 \%$ & $82 \%$ & $65 \%$ & $19 \%$ & $18 \%$ & $35 \%$ \\
\hline SENEGAL & $88 \%$ & $100 \%$ & $100 \%$ & $12 \%$ & $0 \%$ & $0 \%$ \\
\hline MADAGASCAR & $86 \%$ & $70 \%$ & $60 \%$ & $14 \%$ & $30 \%$ & $40 \%$ \\
\hline HAUTE-VOLTA & $82 \%$ & $70 \%$ & $80 \%$ & $18 \%$ & $30 \%$ & $20 \%$ \\
\hline MARTINIQUE & $13 \%$ & $13 \%$ & $13 \%$ & $87 \%$ & $87 \%$ & $87 \%$ \\
\hline $\begin{array}{c}\text { GUADELOUPE } \\
1\end{array}$ & $0 \%$ & $37 \%$ & $17 \%$ & $100 \%$ & $63 \%$ & $83 \%$ \\
\hline \begin{tabular}{|c|} 
GUADELOUPE \\
II
\end{tabular} & $18 \%$ & $16 \%$ & $21 \%$ & $82 \%$ & $84 \%$ & $79 \%$ \\
\hline PORTO-RICO & $23 \%$ & $18 \%$ & $0 \%$ & $77 \%$ & $82 \%$ & $100 \%$ \\
\hline BRESIL & $28 \%$ & $0 \%$ & $0 \%$ & $72 \%$ & $100 \%$ & $100 \%$ \\
\hline
\end{tabular}

Fig. 1. Chétotaxie comparée de neuf souches de Schistosoma mansoni. Les sensilles prises en considération pour la diagnose sont en noir, les autres en blanc. 
Le total des papilles préfurcales ventrales et dorsales chez les cercaires africaines est le plus souvent de quatre à cinq papilles; il est, le plus souvent, de six ou plus de six papilles chez les cercaires américaines.

Aucune souche, toutefois, n'est entièrement homogène pour les trois caractères différentiels observés; dans chaque souche africaine, nous avons trouvé un petit pourcentage de cercaires ayant soit des $\mathrm{A}_{1} \mathrm{~L}-\mathrm{A}_{11} \mathrm{~L}$ alignées, soit trois préfurcales ventrales, soit trois préfurcales dorsales; de même, chaque souche américaine a montré un petit nombre de cercaires avec des $\mathrm{A}_{1} \mathrm{~L}-\mathrm{A}_{11} \mathrm{~L}$ disposées en triangle, ou deux préfurcales ventrales, ou deux préfurcales dorsales. La figure 1 présente les proportions respectives observées pour chaque souche. Il n'y a pas de différence absolue entre les cercaires des deux continents, et nous n'avons observé, dans les souches américaines, aucune particularité chétotaxique nouvelle : il s'agit toujours de l'espèce Schistosoma mansoni. Toutefois, nous distinguons la chétotaxie d'une souche américaine passant par Biomphalaria glabrata de celle d'une souche africaine passant par B. pfeifferi.

Les mollusques émettant ces divers lots de cercaires, c'est-à-dire B. pfeifferi et B. glabrata ont été élevés d'une manière identique, à la même température : $23^{\circ} \mathrm{C}$, dans une eau de même provenance et avec le même type d'aliment : de la laitue ; les variations chétotaxiques observées ne peuvent donc pas être dues à un environnement différent.
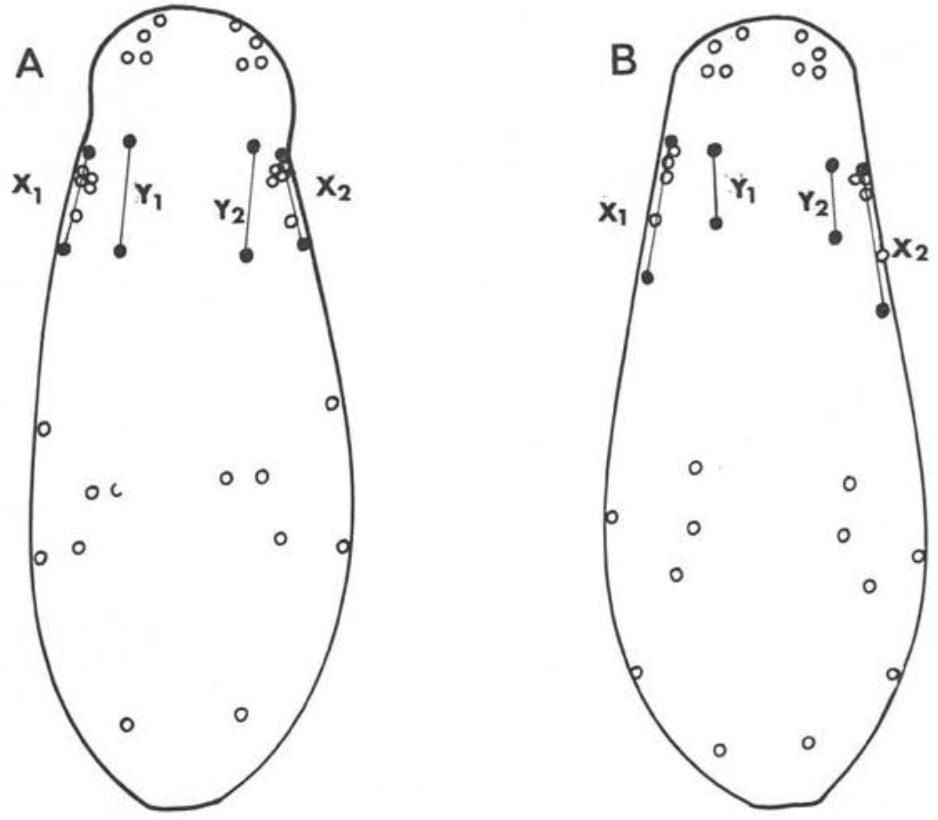

Fig. 2. En noir, soies anté-acétabulaires dorsales et latérales de $S$. mansoni utilisées pour établir l'indice $n$. $x 1$ et $x 2$ : distances entre les papilles $\mathrm{A}_{\mathrm{I}} \mathrm{L}$ et $\mathrm{A}_{11} \mathrm{~L}$; $y 1$ et $y 2$ : distances entre les papilles $\mathrm{A}_{1} \mathrm{D}$ et $\mathrm{A}_{11} \mathrm{D}$. A : type humain ; $\mathrm{B}$ : type murin. 


\section{2. - Soies antéacétabulaires : rapport des distances $A_{I} L-A_{I I} L / A_{I} D-A_{I I} D$.}

A la suite de ces premiers résultats, nous avons tenté d'approfondir le problème par l'utilisation de données numériques et avons choisi pour test le rapport des distances entre les papilles $\mathrm{A}_{\mathrm{I}} \mathrm{L}-\mathrm{A}_{\mathrm{II}} \mathrm{L}$ et $\mathrm{A}_{\mathrm{I}} \mathrm{D}-\mathrm{A}_{\mathrm{II}} \mathrm{D}$ (Ch. Bayssade-Dufour, $1977 \mathrm{~b}$ ) ; la disposition des papilles est représentée sur la figure 2. En appelant $x 1$ et $x 2$ la distance comprise entre la première papille $A_{1} L$ et la papille $A_{11} L$ pour l'un et l'autre hémicorps, $y 1$ et $y 2$ la distance comprise entre la papille $\mathrm{A}_{\mathrm{I}} \mathrm{D}$ et la papille $\mathrm{A}_{\mathrm{II}} \mathrm{D}$ pour l'un et l'autre hémicorps, nous calculons, pour chaque cercaire, un indice donné par la formule :

$$
n=\frac{1}{2} \frac{x 1}{y 1}+\frac{x 2}{y 2} .
$$

Nous avons disposé de trois lots d'œufs de Schistosomes provenant de malades d'Afrique occidentale, et d'un de Madagascar; ils se sont développés tous les quatre chez Biomphalaria pfeifferi. Un lot, provenant d'un malade d'Egypte, s'est développé chez $B$. alexandrina; quatre lots, provenant de malades des Antilles, se sont développés chez B. glabrata.

Les résultats obtenus sont les suivants :

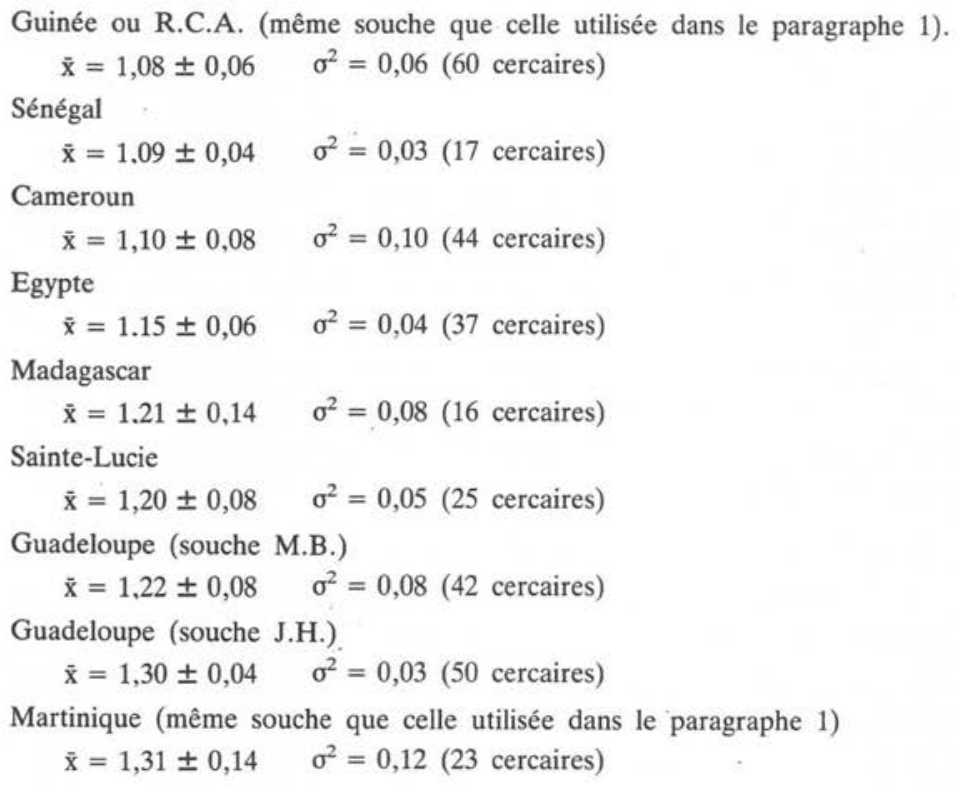

Nous constatons que les cercaires d'Afrique Occidentale passant par B. pfeifferi, et la souche d'Egypte passant par B. alexandrina, ont un indice un peu plus faible que 
celui des cercaires des Antilles passant par B. glabrata. La souche de Madagascar passant par B. pfeifferi a un indice supérieur à celui des autres souches africaines (fig. 3).
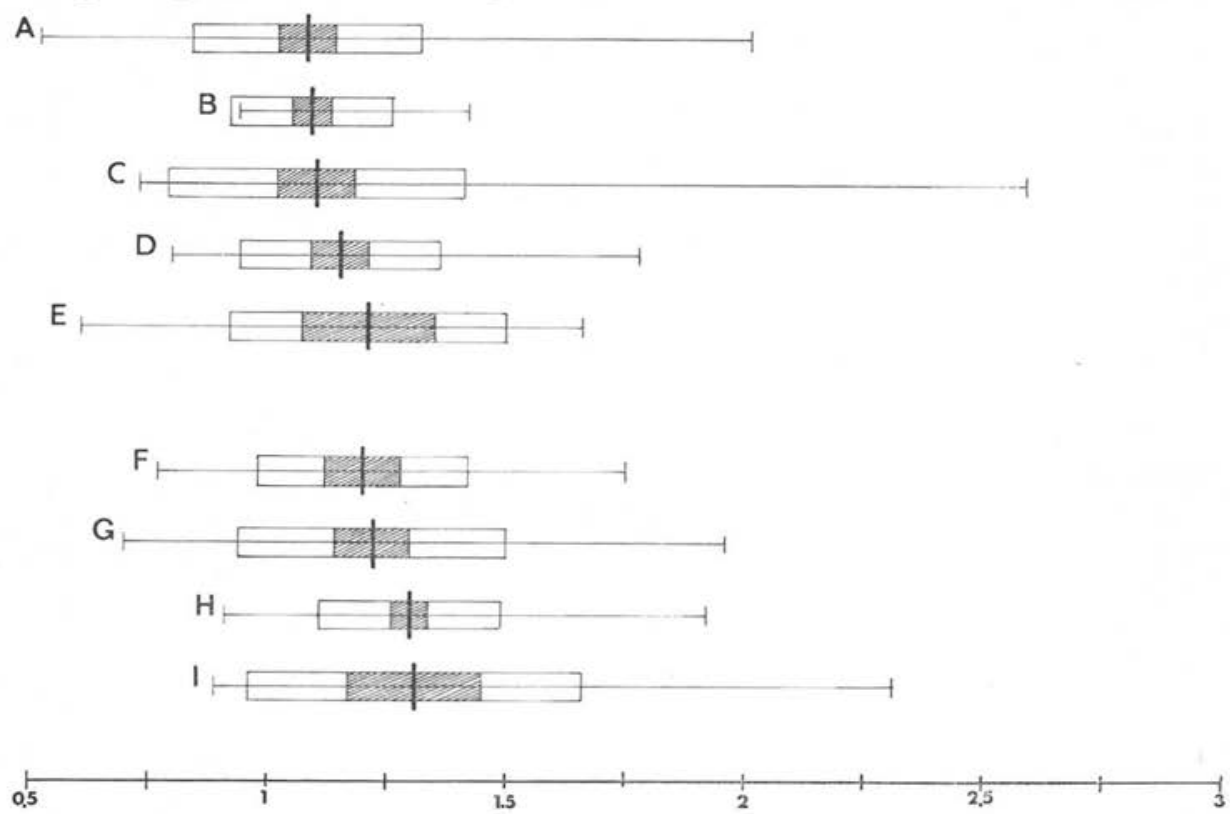

Fig. 3. Indice $n$ des cercaires issues de Biomphalaria pfeifferi (A-C, E), B. alexandrina (D) et B. glabrata (F-I) ; Mollusques infestés à partir de malades africains : A : Guinée-R.C.A. ; B : Sénégal ; C : Cameroun; D : Egypte ; E : Madagascar ; Mollusques infestés à partir de malades américains : F : Sainte-Lucie ; G : Guadeloupe (M.B.) ; H : Guadeloupe (J.H.) ; I : Martinique. Trait vertical : moyenne de l'échantillon ; rectangle hachuré : intervalle de confiance de la moyenne ; rectangle blanc : écart-type de l'échantillon ; ligne horizontale : extrêmes observés.

Pour savoir si les faibles différences constatées sont liées à l'espèce du mollusque ou à un autre facteur, quelques expériences ont été réalisées en utilisant comme hôte intermédiaire le mollusque africain pour les souches américaines et inversement. Les résultats sont les suivants :

- La souche du Sénégal dont l'indice sur $B$. pfeifferi est de $\overline{\mathrm{x}}=1,09 \pm 0,04$ a été également transmise à B. glabrata; dans ce $2^{\mathrm{e}}$ cas, les cercaires émises ont eu pour indice : $\overline{\mathrm{x}}=1,07 \pm 0,08$ (mesures prises sur 12 cercaires).

- La souche de Guadeloupe dont l'indice sur B. glabrata est de $\overline{\mathrm{x}}=1,22 \pm 0,08$ a été transmise également à $B$. pfeifferi; dans ce $2^{\mathrm{e}}$ cas, les cercaires émises ont eu pour indice : $\overline{\mathbf{x}}=1,29 \pm 0,1$ (mesures prises sur 23 cercaires).

- La souche Guinée ou R.C.A., après deux passages successifs B. pfeifferi Souris blanches, a été transmise, lors du $3^{\mathrm{e}}$ passage, d'une part à $B$. pfeifferi, d'autre part à $B$. glabrata ; les indices obtenus sont égaux à : $\overline{\mathrm{x}}=1,30 \pm 0,04$ (mesures prises sur 123 cercaires passées par B. pfeifferi) et $\overline{\mathrm{x}}=1,35 \pm 0,06$ (mesures prises sur 40 cercaires passées par $B$. glabrata). 


\section{Variations selon le vertébré - hôte}

L'indice $n$ ne varie pas avec l'espèce du mollusque, tout au moins lors du premier passage ; les faibles différences constatées à ce point de vue entre souches africaines et souches américaines sont peut-être induites par d'autres facteurs et, en particulier, comme nous allons le voir, par l'intervention éventuelle des infections murines.

Les soies latérales corporelles et les soies préfurcales n'ont pas montré de différence importante entre les souches murines et les souches humaines; nous avons au contraire choisi l'indice $n$, défini dans le paragraphe 2, parce qu'il paraissait être différent dans les souches humaines et les souches murines.

Les résultats obtenus sont les suivants :

a. Souches humaines.

Souches d'Afrique occidentale après un passage chez B. pfeifferi :

$\overline{\mathrm{x}}=1,08 \pm 0,06$.

Souches des Antilles après un passage chez $B$. glabrata :

$\overline{\mathrm{x}}=1,26 \pm 0,08$.

b. Souches murines:

Souche "Brumpt» originaire de Recife, au Brésil, et entretenue depuis 20 ans environ sur B. glabrata et Souris blanche, et occasionnellement sur Hamster :

$$
\overline{\mathrm{x}}=1,77 \pm 0,20 \text {. }
$$

Souche "Pasteur » originaire de Porto-Rico, entretenue durant 10 ans sur Hamster et ensuite, durant deux ans, sur Souris blanche :

$$
\overline{\mathbf{x}}=2,34 \pm 0,22 \text {. }
$$

Souche isolée d'un Rattus rattus capturé à Grand-Etang, Guadeloupe, et passée par un B. glabrata:

$$
\overline{\mathrm{x}}=1,63 \pm 0,06 \text {. }
$$

Souche isolée à partir de six de $B$. glabrata naturellement infestés, récoltés à Grand-Etang, Guadeloupe :

$$
\overline{\mathrm{x}}=1,63 \pm 0,06 \text {. }
$$

Souche «Brumpt» originaire de Recife et ayant subi trois transplants de sporocystes de $B$. glabrata à $B$. glabrata, sans passage sur Souris (expérience J. Jourdane) :

$$
\overline{\mathrm{x}}=1,69 \pm 0,24 \text {. }
$$

Nous constatons donc une différence nette et constante entre l'indice des souches humaines et l'indice des souches murines. 

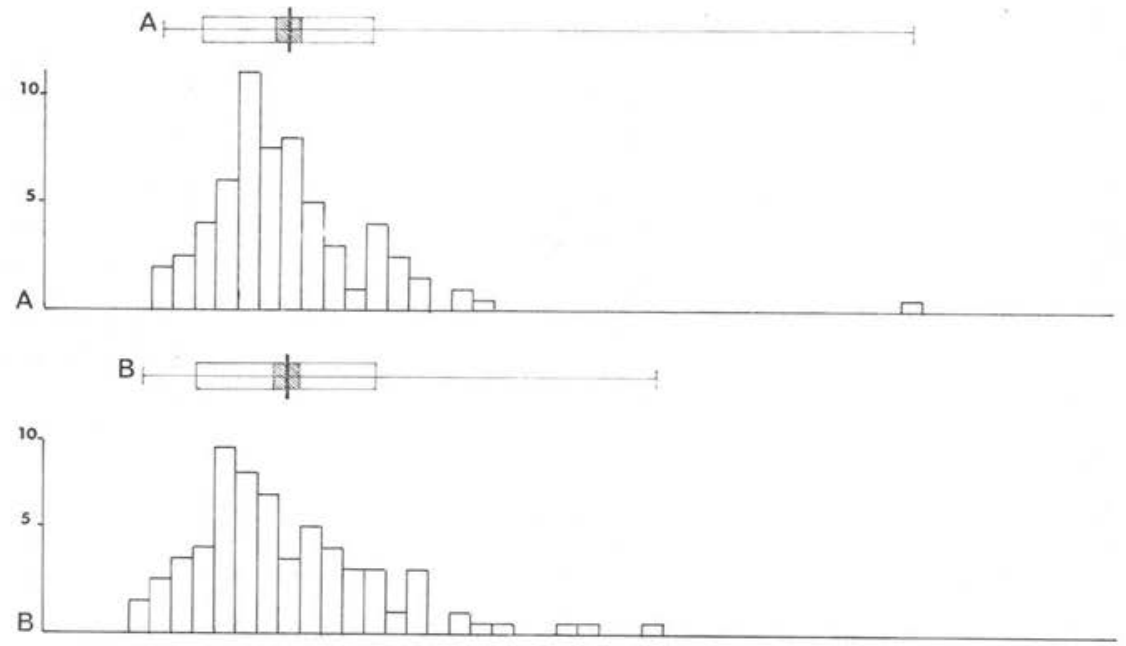

\section{$\mathrm{C}$}
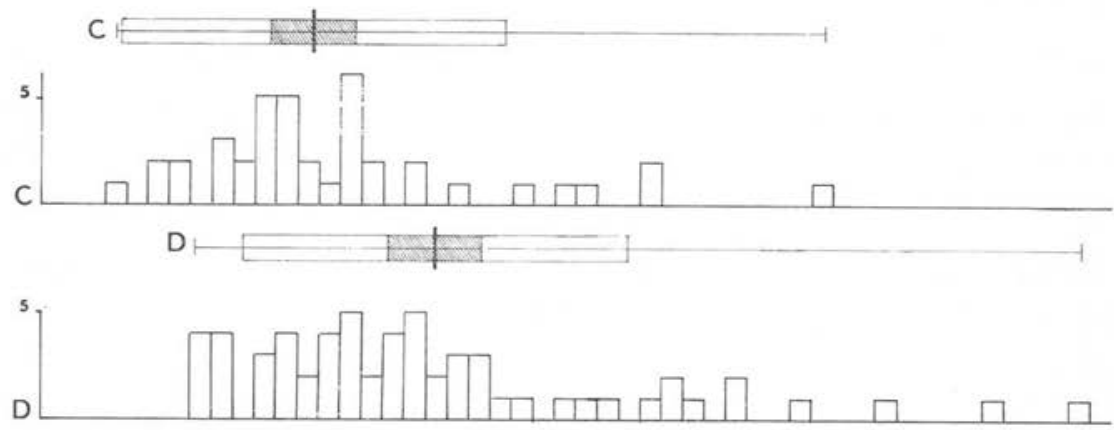

os

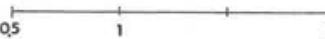

2

4

Fig. 4. Indice des cercaires de la souche Grand-Etang et des cercaires de souches de laboratoire adaptés à la Souris. A : B. glabrata infesté à partir d'un $R$. rattus de la station Grand-Etang ; B : cercaires issues de six B. glabrata de la même station ; C : souche " Brumpt "; D : souche " Pasteur ». En haut, caractéristiques de l'échantillon ; en bas : histogramme ; en abcisses : fréquence de classe des cercaires; en ordonnées : indice $n$. 


\section{Données expérimentales sur l'indice $n$}

Une série d'expériences a été entreprise (Cassone, Bayssade-Dufour, Albaret et Jourdane, $1978 a, 1978 b$ ) pour tenter d'élucider ce phénomène.

Les résultats d'une première série d'expériences sont actuellement disponibles. La souche Guinée-R.C.A. (A) est passée 4 fois par B. pfeifferi et Souris blanche (B.C.D.E.). Les indices des cercaires ont été mesurés à l'origine (A) et à chacun des passages (B.C.D.E.) ; les résultats sont donnés dans le tableau $\mathrm{I}$ et sur la figure 5.

Tableau I

\begin{tabular}{clllll}
\hline & A & B & C & D & E 1 \\
\hline$\mu$ & 1,08 & 1,07 & 1,05 & 1,30 & 1,63 \\
$\sigma^{2}$ & 0,060 & 0,072 & 0,024 & 0,058 & 0,16 \\
$2 \mathrm{~S}$ & 0,06 & 0,06 & 0,04 & 0,04 & 0,10 \\
$\mathrm{~N}$ & 60 & 60 & 60 & 123 & 60 \\
\hline
\end{tabular}

$\mathrm{N}$ indique le nombre de cercaires observés.

Lors du quatrième passage $\mathrm{E}$, nous avons mesuré des indices sur les cercaires issues de 6 Mollusques différents dont les résultats sont consignés dans le tableau II et sur la figure 6 .

Tableau II

\begin{tabular}{ccccccc}
\hline & E 1 & E 2 & E 3 & E 4 & E 5 & E 6 \\
\hline$\mu$ & 1,63 & 1,27 & 1,53 & 1,33 & 1,30 & 1,45 \\
$\sigma^{2}$ & 0,16 & 0,14 & 0,07 & 0,08 & 0,07 & 0,19 \\
$2 \mathrm{~S}$ & 0,10 & 0,10 & 0,06 & 0,06 & 0,06 & 0,01 \\
$\mathrm{~N}$ & 60 & 53 & 54 & 60 & 46 & 60 \\
\hline
\end{tabular}

Un test d'ajustement à une loi normale montre que les populations $\mathrm{A}, \mathrm{B}, \mathrm{C}$, ont une distribution gaussienne avec un $\chi^{2}$ dont la valeur est largement en-deçà de la limite de probabilité d'erreur de $5 \%$. En revanche, l'hypothèse d'une distribution gaussienne pour les populations $\mathrm{D}$ et $\mathrm{E}$ est à rejeter : le $\chi^{2}$ est largement au-delà de la limite d'erreur de $5 \%$ fixée. Ceci montre que ces dernières populations sont significativement différentes des trois autres dont elles sont issues.

En appliquant un test de comparaison de variance (test $\mathrm{F}$ ) aux trois premières populations, on montre que A et B ne diffèrent pas significativement, alors que C diffère significativement des deux autres. On remarque, en effet, sur cette dernière population un resserrement de la base, avec élimination des extrêmes, du moins dans l'effectif de population envisagée (fig. 5). 

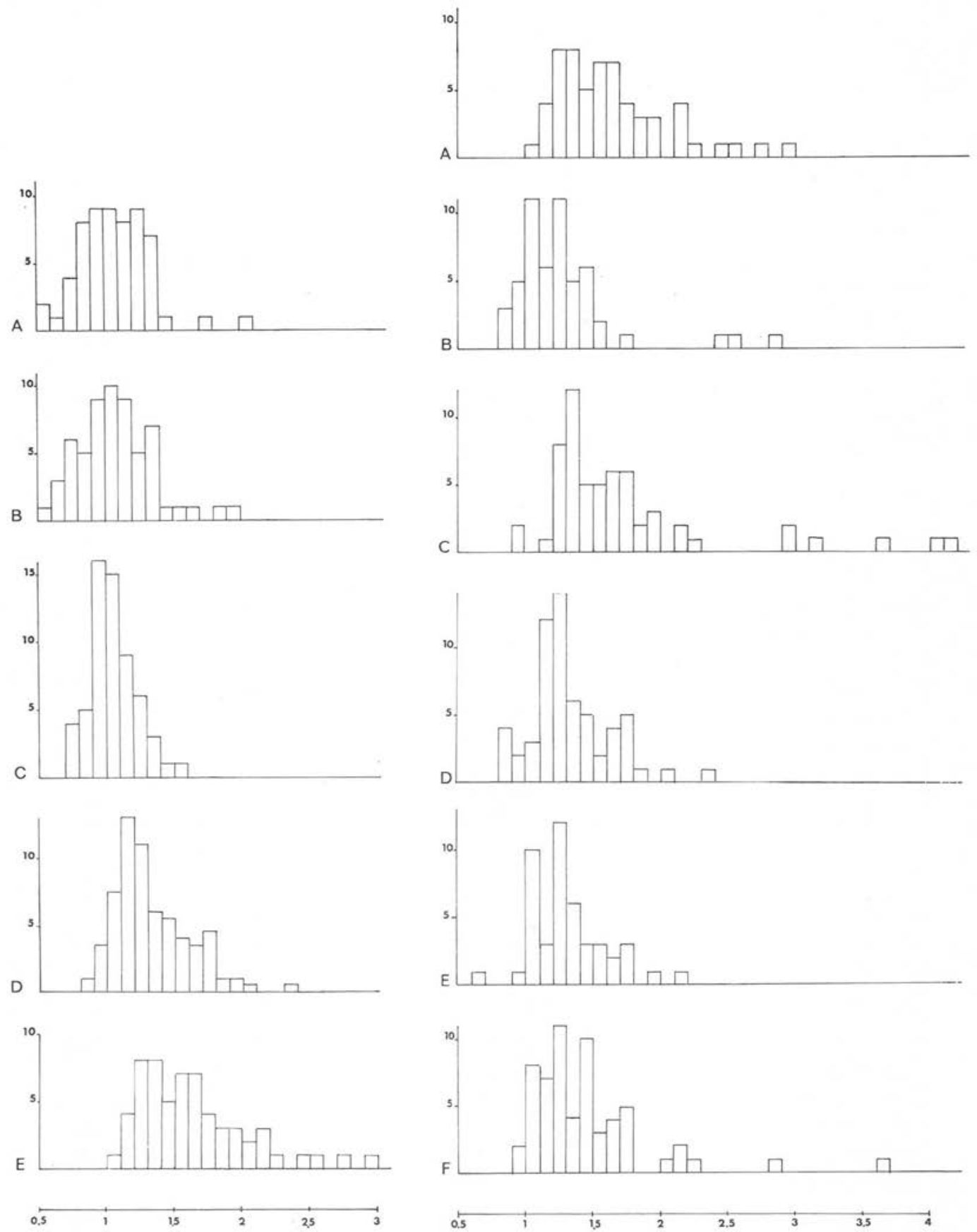

Fig. 5. Adaptation d'une souche humaine à la Souris blanche : A souche humaine "Guinée ou R.C.A. " B, C, D, E : $1^{\mathrm{er}}, 2^{\mathrm{e}}, 3^{\mathrm{e}}, 4^{\mathrm{e}}$ passage par $B$. pfeifferi-Souris blanche.

$\mathrm{A}: \overline{\mathrm{x}}=1,08 ; \mathrm{B}: \overline{\mathrm{x}}=1,07 ; \mathrm{C}: \overline{\mathrm{x}}=1,05 ; \mathrm{D}:$ $\tilde{\mathrm{x}}=1,30 ; \mathrm{E}: \tilde{\mathrm{x}}=1,63$.

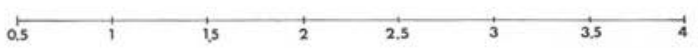

Fig. 6. Quatrième passage d'une souche humaine à la Souris blanche. Histogrammes de chacun des six Mollusques étudiés.

$\mathrm{A}(\mathrm{E} 1): \overline{\mathrm{x}}=1,63 ; \mathrm{B}(\mathrm{E} 2): \overline{\mathrm{x}}=1,27 ; \mathrm{C}(\mathrm{E} 3)$ :

$\overline{\mathrm{x}}=1,53 ; \mathrm{D}(\mathrm{E} 4): \overline{\mathrm{x}}=1,33 ; \mathrm{E}(\mathrm{E} 5):$ $\overline{\mathrm{x}}=1,30 ; \mathrm{F}(\mathrm{E} 6): \overline{\mathrm{x}}=1,45$. 
Analyse des courbes.

L'analyse des courbes obtenues indique que la dispersion des mesures la moins importante s'observe au cours du $2^{\mathrm{c}}$ passage.

Aux $3^{\mathrm{e}}$ et $4^{\mathrm{e}}$ passages, la distribution est asymétrique, avec augmentation du nombre d'individus qui ont un indice fort. Le fait que les histogrammes des $3^{\mathrm{e}}$ et $4^{\mathrm{e}}$ passages ne soient pas ajustables à une loi normale n'est pas incompatible avec l'hypothèse d'un mélange, pour chacun d'eux, de deux morphes distincts :

- pour le $3^{\mathrm{e}}$ passage, il pourrait y avoir deux moyennes : l'une à 1,0 , semblable à celle des souches humaines africaines, l'autre à 1,7 , semblable à celle des souches entretenues depuis de nombreuses générations sur la Souris ;

- au $4^{\mathrm{e}}$ passage, il pourrait y avoir une moyenne aux alentours de 1,3, semblable à celle que l'on observe chez la plupart des souches humaines américaines, et une moyenne à 1,7 , correspondant à celle des souches murines de laboratoire.

Avant de tenter l'interprétation de cette expérience, il nous semble nécessaire d'exposer toute une série de données obtenues par différents collègues, ou par nousmême, qui pourraient aider à la compréhension du phénomène :

\section{Données complémentaires sur l'adaptation des souches humaines aux souris blanches}

\section{1. - Variations chez les miracidiums.}

Albaret, 1978, Albaret et Léger, 1978, travaillant, le plus souvent, sur les mêmes souches que les nôtres, ont montré que le nombre de papilles ciliées au niveau de la ceinture moyenne des miracidiums varie selon l'hôte définitif et selon l'hôte vecteur (fig. 7). Ils distinguent :

- Un premier groupe de miracidiums, ayant un nombre de soies élevé qui caractérise les souches africaines humaines passant par B. pfeifferi.

- Un deuxième groupe, à nombre de soies moins élevé, caractéristique des souches humaines américaines passant par B. glabrata.

- Les miracidiums de souches humaines, après un passage chez la Souris, ont un nombre de soies qui diminue brusquement, et constituent un troisième groupe.

- Un quatrième groupe comprend les souches murines de Guadeloupe d'une provenance autre que Grand-Etang ; leurs miracidiums ont un nombre de soies très réduit.

- La souche murine de Grand-Etang et la souche Brumpt se situent entre le $2^{\mathrm{c}}$ et le $3^{\mathrm{e}}$ groupe. 
$1^{e r}$ groupe: souches humaines africaines:

Guinée - R.C.A.......................

Madagascar

Cameroun

Soudan

Sénégal

$2^{e}$ groupe: souches humaines américaines:

Martinique

Guadeloupe (souche M.B.)

$$
\begin{array}{cc}
m & 2 S \\
\overline{\mathrm{x}}=23,16 \pm 0,50 \\
\overline{\mathrm{x}}=23,03 \pm 0,38 \\
\overline{\mathrm{x}}=23,02 \pm 0,44 \\
\overline{\mathrm{x}}=22,83 \pm 0,62 \\
\overline{\mathrm{x}}=22,26 \pm 0,30
\end{array}
$$
$\sigma^{2}$ 1,96 2,14

$\tilde{\mathrm{x}}=21,56 \pm 0,28$

$\overline{\mathrm{x}}=21,22 \pm 0,32$

Groupe intermédiaire: souches intermédiaires adaptées aux murins :

souche Brumpt

$\overline{\mathrm{x}}=21,02 \pm 0,44$

souche Grand-Etang

$\overline{\mathrm{x}}=19,84 \pm 0,64$

$3^{e}$ groupe: souche humaine après un passage sur souris :

Guinée - R.C.A.

$$
\overline{\mathrm{x}}=19,93 \pm 0,46
$$

$4^{e}$ groupe: souches murines de Guadeloupe autres que Grand-Etang :

Rat Marigot

$$
\overline{\mathrm{x}}=17,64 \pm 1,16
$$

$\overline{\mathrm{x}}=17,28 \pm 0,80$

Le nombre de soies semble être en corrélation avec la longueur du miracidium.

Les résultats d'Albaret et Léger sur les miracidiums paraissent donc plus faciles à interpréter que ceux que nous obtenons sur les cercaires; le nombre de soies (et la taille du miracidium) semble traduire le niveau d'adaptation entre le parasite et ses hôtes successifs : B. pfeifferi est meilleur hôte que B. glabrata; 1'Homme est meilleur hôte que la Souris, qui est elle-même meilleur hôte que ne l'est le Rat. Les miracidiums de la souche de Grand-Etang paraissent beaucoup mieux adaptés au Rat que

\begin{tabular}{|c|c|c|}
\hline & $2 S$ & $\sigma^{2}$ \\
\hline miracidiums humains & $\overline{\mathrm{x}}=23,16 \pm 0,50$ & 1,96 \\
\hline r passage sur souris & $\overline{\mathrm{x}}=19,93 \pm 0,46$ & 3,51 \\
\hline ixième passage sur souris .... & $\overline{\mathrm{x}}=19,70 \pm 0,46$ & 2,8 \\
\hline isième passage sur souris .................................... & $\overline{\mathrm{x}}=19,55 \pm 0,50$ & 2,2 \\
\hline trième passage sur souris & $\overline{\mathrm{x}}=20,34 \pm 0,44$ & 2,5 \\
\hline nquième passage sur souris & $\overline{\mathrm{x}}=20,92 \pm 0,66$ & 4,7 \\
\hline
\end{tabular}
ne le sont les miracidiums des Rats d'autres localités de Guadeloupe.

L'hypothèse de la corrélation entre le nombre de papilles ciliées et le degré d'adaptation du parasite à son hôte vertébré semble bien rendre compte des résultats obtenus sur le miracidium.

Lorsque Albaret et Léger, parallèlement à nos expériences sur les cercaires, étudient le nombre de papilles des miracidiums de la souche Guinée-R.C.A. lors de passages successifs par B. pfeifferi - Souris blanche, leurs résultats sont les suivants :

Le résultat du cinquième passage ressemble beaucoup à celui de la souche Brumpt adaptée depuis de nombreuses années aux souris où $\bar{x}=21,02 \pm 0,44$. 
Nous trouvons ici la notion d'une adaptation à la Souris se déclenchant très tardivement après le $3^{e}$ passage ; le même phénomène apparaît, nous allons le voir, dans d'autres données objectives.
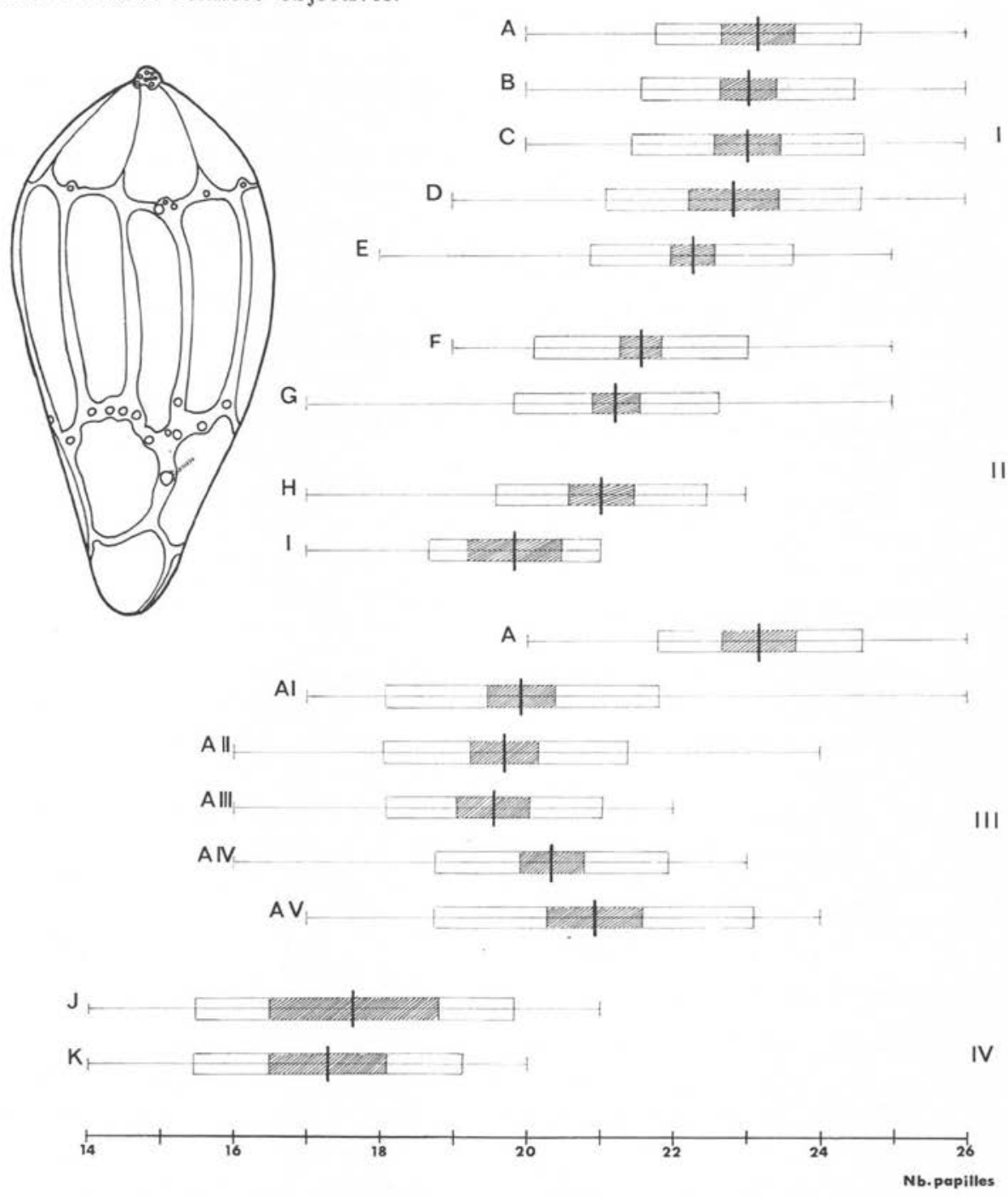

Fig. 7. Variation du nombre des papilles ciliées chez les miracidiums de $S$. mansoni (expérience Albaret-Léger). - Homme-Afrique : $\mathrm{A}=$ Guinée-R.C.A. ; $\mathrm{B}=$ Madagascar ; $\mathrm{C}=$ Cameroun ; $\mathrm{D}=$ Soudan $; \mathrm{E}=$ Sénégal ; Homme-Amérique : $\mathrm{F}=$ Martinique ; $\mathrm{G}=$ Guadeloupe (M.B.) ; - Souches adaptées aux Muridés : $\mathrm{H}=$ « Brumpt »; $\mathrm{I}=$ " Grand-Etang » ; Passage Homme-Souris : $A=$ Guinée-R.C.A. ; $A_{I}, A_{I I}, A_{I I I}, A_{I V}, A_{V}: 1^{\mathrm{er}}, 2^{\mathrm{e}}, 3^{\mathrm{e}}, 4^{\mathrm{e}}$ et $5^{\mathrm{e}}$ passages B. pfeifferi-Souris blanche ; Rats Guadeloupe : $\mathrm{J}=$ Marigot ; $\mathrm{K}=$ Gosier. 


\section{2. - Réussite des passages expérimentaux.}

Les expérimentateurs (1) s'accordent à constater qu'une souche humaine s'adapte aisément au cours du $1^{\text {er }}$ et du $2^{\mathrm{e}}$ passage aux conditions expérimentales. Plus de $50 \%$ des Mollusques s'infestent facilement, alors que, lors du $3^{\mathrm{e}}$ et du $4^{\mathrm{e}}$ passage, la plupart des Mollusques exposés aux miracidiums issus de la $2^{\circ}$ et de la $3^{\mathrm{e}}$ génération restent négatifs ; il est fréquent de perdre la souche à ce moment-là. Dans un troisième temps, au contraire, lors des $5^{\mathrm{e}}$ et $6^{\mathrm{e}}$ passages, la souche paraît s'adapter à la Souris, et les passages successifs redeviennent aisés.

Lors de l'expérience concernant l'adaptation de la souche Guinée-R.C.A. à la Souris blanche, nos données numériques sont les suivantes:

$-1^{\text {re }}$ infestation : 3 Mollusques infestés : 3 positifs $-100 \%$ de réussite ;

$-2^{\mathrm{e}}$ infestation, après 1 passage sur souris : 11 Mollusques infestés : 6 positifs $54 \%$ de réussite ;

- $3^{\mathrm{e}}$ infestation, après 2 passages sur souris : 38 Mollusques infestés : 3 positifs $7 \%$ de réussite ;

$-4^{\mathrm{e}}$ infestation, après 3 passages sur souris : 24 Mollusques infestés : 6 positifs $25 \%$ de réussite.

\section{3. - Durée de la prépatence.}

A $23{ }^{\circ} \mathrm{C}$, dans une pièce à luminosité réduite, la prépatence des Mollusques varie ; elle est comprise entre 35 et 40 jours pour les infestations effectuées à partir de miracidiums humains et pour les infestations effectuées après un ou deux passages sur la Souris.

Après trois passages sur Souris, pour la souche Guinée-R.C.A., nous avons observé une prépatence de 43 jours et, après quatre passages sur Souris, pour la même souche, la prépatence observée pour les six Mollusques qui ont été positifs s'est étalée entre 49 et 63 jours.

\section{4. - Traversée de la peau.}

En collaboration avec J. Bénex (1978), nous avons refait l'expérience de Clegg et Smithers, 1971, qui consiste à faire passer des cercaires à travers un fragment de peau de Rat pour obtenir des Schistosomules, et nous avons obtenu les résultats suivants :

- des cercaires de souches murines traversent la peau du Rat et la transformation en schistosomules est de l'ordre de $20 \%$.

(1) Nous remercions nos collègues Claude Combes, René Houin, Jacques Lapierre et Hugues Picot, de nous avoir fait part de leurs remarques sur ce point. 
- des cercaires de souches humaines africaines (Sénégal, Cameroun), ou américaines (Guadeloupe), ne traversent qu'en nombre très faible et la transformation en schistosomules est inférieure ou égale à $1 \%$.

La contre-expérience a été réalisée avec un fragment de peau humaine; les résultats ont été inversés, les cercaires provenant de cycles passant par des murins n'ont pratiquement pas traversé la peau, et le pourcentage observé de transformation en schistosomules a été extrêmement faible, voisin de zéro. L'expérience concernant les cercaires de souches humaines n'a pas donné de bons résultats (peau mal dégraissée), mais ceux-ci sont cependant supérieurs aux précédents (5\% environ).

\section{5. - Localisation du parasite.}

Lancastre, Nassi et Poirot, 1976, ont mis en évidence une localisation pulmonaire massive des Schistosomes adultes chez $R$. norvegicus de Guadeloupe, qui s'oppose aux localisations hépatiques et mésentériques précédemment décrites.

\section{6. - Fécondité du parasite.}

Une recherche systématique destinée à obtenir des données numériques précises n’a pas encore été effectuée. Il est possible cependant d'indiquer que les œufs trouvés dans les selles humaines sont viables dans une très forte proportion; les œufs trouvés chez les Rats spontanément infestés, récoltés en Guadeloupe sont peu nombreux et un faible pourcentage seulement (moins de $5 \%$ ) va permettre l'éclosion d'un miracidium.

Dans le seul cas observé d'un Rat de Grand-Etang, nous avons eu l'impression, au contraire, que les œufs étaient nombreux et régulièrement fertiles; il en est de même pour les souches «Brumpt» (Brésil) et «Pasteur» (Porto-Rico), adaptées depuis longtemps à la Souris.

\section{7. - Données immunologiques.}

Par l'étude de la réponse immunitaire, N. Léger et coll., 1976, ont montré la présence en Guadeloupe d'un système «Rat-Schistosome » particulièrement bien adapté (faible réaction immunologique, persistance de l'infestation) favorable à l'entretien de la parasitose. Ce résultat s'oppose à ce qui est obtenu pour les autres couples « Rat-Schistosomes » expérimentés.

\section{Tentatives d'interprétation}

L'ensemble de ces données diverses nous indique donc que Schistosoma mansoni est un parasite humain particulièrement bien adapté à Biomphalaria pfeifferi; il paraît un peu moins bien adapté au Mollusque américain B. glabrata. L'adaptation de l'espèce aux muridés constitue un phénomène complexe, car la sélection ne s'ef- 
fectue pas au premier passage, comme il est habituel lorsqu'on cherche à adapter un parasite à un hôte anormal ; cette adaptation nécessite 5 ou 6 passages successifs et il semble que ce soit au cours de la $3^{\mathrm{e}}$ ou $4^{\mathrm{e}}$ génération seulement que se manifeste l'essentiel du phénomène.

Le phénomène génétique devant lequel nous nous trouvons reste mystérieux. Dans les expériences de transformation d'une souche africaine d'indice 1,0 en une souche murine d'indice 1,7 , nous pouvions nous attendre à voir une population bimodale autour de ces deux valeurs, le morphe à 1,0 se vidant progressivement tandis que le morphe à 1,7 prendrait de l'importance. L'expérience $\mathrm{E}$ ( $4^{\mathrm{e}}$ passage) semble indiquer au contraire qu'il $\mathrm{y}$ a un morphe à 1,3 et un morphe à 1,7 .

Cette constatation nous amène donc à formuler l'hypothèse selon laquelle l'indice 1,3 ne serait pas la résultante de l'addition de 2 courbes, mais, au contraire, un palier d'équilibre biologique. L'indice des soies anté-acétabulaires de $S$. mansoni pourrait, dans cette hypothèse, avoir 3 types d'équilibre : 1,0,1,3 et 1,7. Le palier à 1,3 pourrait se retrouver chez toutes les souches qui passent de façon irrégulière tantôt chez l'Homme, tantôt chez le Rat, quel que soit le nombre précis de passages chez l'un et chez l'autre. On comprendrait ainsi plus facilement pourquoi presque tous les indices rencontrés dans les infections naturelles de Guadeloupe se situent autour de 1,3 .

\section{Données épidémiologiques en Guadeloupe}

L'infection des Muridés par Schistosoma mansoni est rarement signalée en Afrique; elle n'est pas exceptionnelle, au contraire, en Amérique, et l'on connaît mal l'importance pratique de ce phénomène (Nassi, Lancastre et Poirot, 1977). Une règle très générale en parasitologie est qu'un parasite mal adapté à son hôte est beaucoup plus pathogène qu'un parasite bien adapté; la question qui se pose, ainsi que le suggère notre collègue $\mathrm{N}$. Léger, est donc de chercher à savoir si les cas très graves de bilharziose intestinale, fréquents au Brésil, et parfois signalés aux Antilles, qui entraînent rapidement la mort du malade par cirrhose portale, ne seraient pas le fait de souches bien adaptées au Rat, réinfestant secondairement l'Homme.

Une autre question importante est de savoir s'il existe des foyers exclusivement murins susceptibles de conserver l'infection, alors même qu'une prophylaxie intensive aurait éradiqué la maladie chez l'Homme.

Les données chétotaxiques paraissent actuellement constituer le meilleur instrument pour aborder ces importants problèmes.

Cassone, Bayssade-Dufour, Albaret et Jourdane, 1978 b, tentent de préciser l'indice de cercaires provenant de Mollusques récoltés en différents biotopes de Guadeloupe; les renseignements sont les suivants : 


\section{1. - Marigot.}

Il s'agit d'une station particulièrement étudiée, où Rioux et coll. (1977) ont montré qu'il s'agissait d'une infection humaine entretenue par un petit nombre de points de contamination intense dans lesquels les Rats s'infestent également. En revanche, lorsqu'on s'éloigne des points de contamination humaine, il n'y a plus de bilharziose, même lorsque Rats et Mollusques cohabitent.

Les cercaires de 21 Mollusques récoltés en ce lieu par Théron et Nassi ont les indices suivants :

$\begin{array}{llll}1,35 \pm 0,1 & 1,44 \pm 0,08 & 1,43 \pm 0,08 & 1,26 \pm 0,12 \\ 1,32 \pm 0,08 & 1,31 \pm 0,06 & 1,11 \pm 0,06 & 1,36 \pm 0,1 \\ 1,22 \pm 0,1 & 1,24 \pm 0,1 & 1,38 \pm 0,14 & 1,31 \pm 0,08 \\ 1,37 \pm 0,1 & 1,38 \pm 0,12 & 1,35 \pm 0,12 & 1,40 \pm 0,2 \\ 1,20 \pm 0,08 & 1,30 \pm 0,06 & 1,58 \pm 0,18 & 1,67 \pm 0,24 \\ 1,39 \pm 0,1 & & & \end{array}$

Nous obtenons donc une moyenne générale de 1,35 , qui correspond bien à l'indice des souches directement isolées à partir de malades guadeloupéens ; nous devons cependant noter qu'à deux reprises, l'indice observé est nettement plus élevé $(1,58$ et 1,67$)$ et les histogrammes correspondants ne s'éloignent pas beaucoup de ceux que l'on obtient expérimentalement lors des $3^{e}$ et $4^{e}$ passages d'une souche humaine à la souris.

\section{2. - Devarieux.}

Les Mollusques ont été récoltés dans un biotope très contaminé par des selles humaines, à proximité de la mangrove où les Rats sont très abondants (Golvan, Houin, Combes, Deniau et Lancastre, 1977).

Les cercaires de 31 Mollusques récoltés par Théron et Nassi ont pour indice :

$\begin{array}{llll}1,47 \pm 0,16 & 1,05 \pm 0,08 & 1,42 \pm 0,12 & 1,24 \pm 0,08 \\ 1,29 \pm 0,06 & 1,30 \pm 0,12 & 1,36 \pm 0,16 & 1,57 \pm 0,14 \\ 1,37 \pm 0,16 & 1,21 \pm 0,06 & 1,27 \pm 0,12 & 1,15 \pm 0,06 \\ 1,25 \pm 0,08 & 1,33 \pm 0,1 & 1,35 \pm 0,18 & 1,50 \pm 0,18 \\ 1,38 \pm 0,14 & 1,23 \pm 0,06 & 1,39 \pm 0,14 & 1,41 \pm 0,1 \\ 1,10 \pm 0,06 & 1,47 \pm 0,16 & 1,32 \pm 0,12 & 1,32 \pm 0,14 \\ 1,24 \pm 0,08 & 1,35 \pm 0,14 & 1,22 \pm 0,1 & 1,24 \pm 0,12 \\ 1,12 \pm 0,1 & 1,33 \pm 0,14 & 1,30 \pm 0,08 & \end{array}$

La moyenne générale se situe à 1,30 et les résultats obtenus sont donc très comparables à ceux de Marigot. 


\section{3. - La grande mangrove.}

La grande mangrove de Guadeloupe est densément peuplée de Rats. Ces Rats ont une durée de vie limitée; selon Delattre, $90 \%$ de la population murine est renouvelée entre mars et octobre.

L'arrière-mangrove est périodiquement inondée; l'eau monte dès la fin du mois d'août et ne se retire qu'en février; or, en septembre, selon Combes, $10 \%$ des Rats seulement sont parasités, tandis qu'à la fin de la période d'inondation, en février, $100 \%$ sont bilharziens; selon Mougeot et Golvan, 1977, lorsque le biotope est en eau, les Rats se réfugient en bordure de mangrove pour aller se nourrir dans une zone de culture où la fréquentation humaine est importante.

Au début de la saison des pluies, selon Pointier et Combes, 1976, aucun Mollusque n'est positif ; un mois et demi après la remise en eau et jusqu'à la fin de la période d'inondation, 2 à 4 pour 1000 Mollusques sont infestés.

Tous ces éléments semblent donc indiquer que les Rats s'infestent essentiellement de septembre à février, à une période de l'année où la mangrove est inondée et où les animaux se réfugient en périphérie, dans la zone de forte fréquentation humaine.

Nous avons pu examiner les cercaires de 6 Mollusques de pleine mangrove; leurs indices sont les suivants:

$$
\begin{array}{lll}
1,43 \pm 0,12 & 1,37 \pm 0,14 & 1,38 \pm 0,06 \\
1,42 \pm 0,08 & 1,21 \pm 0,06 & 1,52 \pm 0,12
\end{array}
$$

La moyenne générale est donc à 1,39 , c'est-à-dire qu'elle est à peine supérieure à celle des foyers humains examinés précédemment.

\section{4. - Grand-Etang.}

Le gîte de Grand-Etang a particulièrement attiré l'attention de Combes, Léger et Golvan, 1975 ; ils écrivent: "Les cercaires de Schistosoma mansoni se rencontrent parfois dans des collections d'eau situées en altitude, dans des lieux peu fréquentés par l'homme, nettement au-dessus du niveau des dernières habitations et des dernières cultures. Ce cas est en particulier celui de Grand-Etang, dans le Sud de l'île montagneuse de Basse-Terre, où $B$. glabrata se révèle parasité par $S$. mansoni tout-au-long de l'année. Cet étang forestier d'altitude est visité par quelques chasseurs ou pêcheurs, voire par de rares touristes, qui, d'ailleurs, doivent se contenter de deux points très limités pour approcher de l'eau, le reste du pourtour étant très difficile d'accès. Ces visites humaines occasionnelles ne nous paraissent pas pouvoir expliquer la constance et la densité de l'infestation de la population locale de Mollusques ». Et, plus loin : "En raison des caractéristiques écologiques du Grand Etang que nous venons brièvement d'indiquer, tout porte à croire que les rats, dans cette zone forestière, sont capables d'assurer la pérennité du foyer de S. mansoni; l'homme n'interviendrait ici que de façon accidentelle ». 
Pour déterminer l'indice des cercaires issues des Mollusques de Grand-Etang, nous avons disposé de 6 spécimens récoltés dans le biotope, mais, à l'époque de cette étude, nous n'isolions pas les cercaires de chacun des Mollusques.

Six Mollusques positifs ont été rassemblés dans un récipient et les cercaires ont été prélevés pendant une quinzaine de jours au fur et à mesure qu'elles étaient émises. Les lames disponibles correspondent donc à des jours d'émission différents et peut-être même à des Mollusques différents ; les indices obtenus varient de façon assez sensible. Une lame a un indice $\overline{\mathrm{x}}=1,49 \pm 0,08$, l'autre lame $1,77 \pm 0,1$; l'indice global est de $n=1,63 \pm 0,06$.

Par ailleurs, une souche isolée d'un Rattus rattus $^{(1)}$ naturellement infesté de Grand-Etang, passée sur un $B$. glabrata, pour indice $\bar{x}=1,63 \pm 0,06$. Il s'agit donc là d'un indice très différent de celui qui était observé dans les gîtes de bilharziose humaine et même dans les gites de bilharziose murine de type mangrove.

Nous ne connaissons des indices comparables que chez des souches adaptées expérimentalement, depuis de très nombreuses années, aux Muridés.

Cela nous conduit logiquement à admettre :

1. que les conclusions de Combes et coll. sur la nature murine de l'infection de GrandEtang sont parfaitement justifiées et que, contrairement à ce qui était admis jusqu’à maintenant, il peut exister des foyers permanents de bilharziose murine sans intervention humaine,

2. qu'une infection même intense des Muridés ne signifie pas nécessairement qu'il s'agisse d'une bilharziose murine; les indices cercariens trouvés en pleine mangrove nous laissent supposer qu'il s'agit d'une infestation mixte ; plusieurs facteurs (migration des Rats, faible infectivité des déjections des Muridés) font que les Mollusques, même lorsqu'ils vivent en pleine mangrove ont peu de chances d'être infestés par des souches ayant eu 7 ou 8 passages successifs chez le Rat sans intervention d'une génération d'origine humaine.

\section{Bibliographie}

Albaret J.-L. (1978) : Variations du nombre des papilles ciliées chez les miracidiums de différentes souches de Schistosoma mansoni, agent de la bilharziose intestinale. C.R. Acad. Sci., 286, sér. D, 1359-1362.

Albaret J.-L., Léger N. (1978): Variations chétotaxiques du miracidium de Schistosoma mansoni selon l'adaptation aux Muridés. Données expérimentales et données épidémiologiques en Guadp. loupe. Ann. Parasitol. Hum. Comp., 53, 617-622.

(1) Rattus norvegicus, bien qu'il ait des biotopes un peu différents, coexiste partout en Guadeloupe avec Rattus rattus et se trouve parfois naturellement infesté. Alors que, dans nos expériences, Rattus rattus s'infeste régulièrement chaque fois qu'il est exposé aux cercaires, nous avons, dans les deux cas expérimentés, eu un échec total avec Rattus norvegicus en Guadeloupe. La question se pose de savoir, si ces expériences se confirmaient, s'il faudrait admettre que $R$. norvegicus ne peut être infesté qu'avec des souches préalablement adaptées au Rat noir. 
Bayssade-Dufour Ch. (1977 a): Différences au niveau des sensilles entre les cercaires africaines et américaines de Schistosomr mansoni, agent de la bilharziose intestinale humaine. C.R. Acad. Sci. Paris, 284, sér. D, 191-193.

Bayssade-Dufour Ch. (1977 b) : Variations chétotaxiques chez les cercaires de Schistosoma mansoni, agent de la bilharziose intestinale, en corrélation avec l'hôte vertébré du parasite. C.R. Acad. Sci. Paris, 285, sér. D : 1511-1513.

Bénex J., Bayssade-Dufour Ch. (1978) : Constatations expérimentales relatives à la spécificité des cercaires de Schistosoma mansoni envers leurs hôtes Mammifères. Ann. Parasitol. Hum. Comp., $53,120$.

Cassone J., Bayssade-Dufour Ch., Albaret J.-L., Jourdane J. (1978 a) : Variations chétotaxiques des cercaires d'une souche humaine de Schistosoma mansoni par passages successifs sur Souris. Ann. Parasitol., Hum. Comp., 53, 387-391.

Cassone J., Bayssade-Dufour Ch., Albaret J.-L., Jourdane J. (1978 b) : L'« indice cercarien " de Schistosoma mansoni. Données expérimentales et épidémiologiques. Ann. Parasitol. Hum. Comp. (en préparation).

Clegg J. A., Smithers S. R. (1971): The effects of immune Rhesus monkey serum on schistosomula of Schistosoma mansoni during cultivation in vitro. Intern. J. Parasitol., 2, 79-98.

Combes C., Léger N., Golvan Y.-I. (1975a) : Le rôle du Rat dans la dynamique de l'endémie schistosomienne en Guadeloupe. C.R. Acad. Sci. Paris, 281, sér. D : 1059-1061.

Golvan Y.-J., Houin R., Combes C., Deniau H., Lancastre F. (1977) : Transmission naturelle de la bilharziose à S. mansoni en Guadeloupe. Ann. Parasitol. Hum. Comp., 52, 259-275.

Lancastre F., Nassi H., Poirot J.-L. (1976) : Sur la localisation pulmonaire massive de S. mansoni chez les Rats (Rattus norvegicus) en Guadeloupe. Ann. Parasitol. Hum. Comp., 51, 345-353.

Léger N., Niel G., Nottheghem M.-J., Forget E. (1976) : Variations de la réponse immunitaire chez divers couples "Rat-bilharzie ». C.R. Acad. Sci. Paris, 283, sér. D : 187-190.

Mougeot G., Golvan Y.-J. (1977): Aspects de la bilharziose murine dans la mangrove douce et l'arrière mangrove de la Grande-Terre en Guadeloupe. Ann. Parasit. Hum. Comp., 52, 623-628.

Nassi H., Lancastre F., Poirot J.-L. (1977) : Rats et bilharziose en Guadeloupe. Nouvelles données. Ann. Parasitol. Hum. Comp., 50, 731-733.

Pointier J.-P., Combes C. (1976) : La saison sèche en Guadeloupe et ses conséquences sur la démographie des Mollusques dans les biotopes à Biomphalaria glabrata (Say, 1818) vecteur de la bilharziose intestinale. Terre, Vie, 30, 121-147.

Richard J. (1971) : La chétotaxie des cercaires. Valeur systématique et phylétique. Mém. Mus. Nat. Hist. Nat., A, Zool., 67, 1-179.

Rioux J.-A., Decamps H., Lanotte G., Combes C., Théron A., Pointier J.-P., Seytor S., Delattre P., Bougerol C. (1977) : Ecologie de la schistosomose intestinale en Guadeloupe. Analyse du système épidémiologique. Documents pour un essai de modélisation. Rev. Epidémiol. Santé Publ., 25, 483-519.

Short R. B., Cartrett M. L. (1973) : Argentophilic papillae of Schistosoma mansoni cercariae. $J$. Parasitol., 59, 1041-1059. 